\title{
Assessment of Refractive Index and Microphysical Parameters of Spherical Aerosols from Data of Dual-Polarization Nephelometer
}

\author{
Christophe Verhaege ${ }^{a}$, Pascal Personne ${ }^{a}$, Valery Shcherbakov ${ }^{\mathrm{a}, \mathrm{b}}$ \\ ${ }^{a}$ Laboratoire de Météorologie Physique, UMR/CNRS 6016, Université Blaise Pascal, 24 avenue des \\ Landais, 63177 Aubière cedex, France \\ ${ }^{b}$ Institute of Physics, Minsk, Belarus
}

\begin{abstract}
It was performed sensitivity tests of dual-polarization polar nephelometer (D2PN) data to optical and microphysical parameters of population of spherical aerosol particles. Measurement errors were modeled as Gaussian random variables with zero mean and a standard deviation of $10 \%$. It is shown that data of the D2PN enable to retrieve microphysical parameters along with the assessment of the refractive index.
\end{abstract}

Key Words: Light scattering; Complex refractive index; Size distribution; Inverse problem; Aerosols

\section{INTRODUCTION}

The knowledge of microphysical and optical characteristics of aerosols is of importance for modeling the radiative balance of Earth's atmosphere, understanding the cloud life cycle, and remote sensing of tropospheric aerosols. Aerosol properties vary widely among various aerosol types (e.g., dust, biomass smoke, urban pollution, sea salt) and geographical regions. A feasible approach to parametrize characteristics of aerosols consists in elaboration of databases. Nowadays a number of light scattering codes are available, often organized in databases. Moreover, a database of experimental results is developed with the light scattering facility in Amsterdam [1].

The long term objective of the D2PN designed at LaMP is to develop a database of optical and microphysical characteristics of aerosols and to test inverse codes against it. The aim of this work is to present results of sensitivity tests of D2PN data to optical and microphysical parameters of populations of spherical aerosol particles.

\section{LABORATORY DUAL-POLARIZATION POLAR NEPHELOMETER (D2PN)}

A diode laser is used as a source of collimated and unpolarized light at the wavelength of $800 \mathrm{~nm}$ and with a power of about $1 \mathrm{~W}$. Two detectors allow measurement of polarized scattered intensities perpendicular $I_{\perp}$ and parallel $I_{/ /}$to the 
scattering plane with the angular resolution of $1^{\circ}$ from $10^{\circ}$ through $169^{\circ}$. The measured light power can range from $10 \mathrm{pW}$ up to $3 \mu \mathrm{W}$. The measurements are quasi-continuous functions of the scattering angle $\theta$. Thus, the degree of linear polarization and the unnormalized phase function can be obtained as functions of $\theta$ within the range between $10^{\circ}$ and $169^{\circ}$. The accuracy of the measurements is estimated to be better than $10 \%$.

\section{SENSITIVITY TESTS}

In our investigations, we use the flexible inverse code [2] and the software package with kernels [3], which were developed by O. Dubovik and colleagues. The software package enables to calculate phase matrix, extinction and absorption for polydisperse spheres and/or randomly oriented oblate and prolate spheroids. The inverse code can be easily adapted to particularities of an experimental set-up. It is built on the principles of statistical estimation, and provides possibility to retrieve particle size distribution along with the assessment of the refractive index value.

Recall that the information content of data, measured by an instrument, has to be sufficient for accurate retrieval of required parameters. In other words, the measured characteristics have to be sensitive enough to variations of the parameters of interest. Otherwise the retrieved values are only a direct consequence of used a priori constraints. Thus, sensitivity tests are indispensable when the inverse code is adapted to a new experimental set-up.

As the first step, we performed the sensitivity tests for the case of populations of spherical aerosol particles. Special attention was paid to the sensitivity of the data of the dual-polarization polar nephelometer to simultaneous variations of the refractive index and microphysical parameters. In the following, the results are analyzed in terms of root mean squared relative errors:

$$
R M S=\frac{1}{n} \sqrt{\sum_{i=1}^{n} \frac{\left(\varphi_{i}(m)-\varphi_{i, \text { meas }}\right)^{2}}{\varphi_{i, \text { meas }}^{2}}}
$$

where $n$ is the number of angles $\theta, \varphi$ is the phase function and $m$ complex refractive index, $\varphi_{\text {meas }}$ corresponds to a synthetic measurement, $\varphi(m)$ is the phase function for the variable refractive index $m=n+\chi i$.

When measurement errors were considered, they were modeled as Gaussian random variables $\varepsilon$ with zero mean and a standard deviation of 0.1 , i.e., of $10 \%$ :

$$
\varphi_{\text {meas }}=\varphi_{\text {comp }}(1+\varepsilon)
$$

where $\varphi_{\text {comp }}$ was computed with input model parameters. The $R M S$ values were computed as the average over 20 realizations.

Size distributions of particles were modeled by a monomodal lognormal function

$$
\frac{d V(r)}{d \ln r}=\frac{C_{V}}{\sqrt{2 \pi \sigma}} \exp \left[-\frac{\left(\ln r-\ln r_{V}\right)^{2}}{2 \sigma^{2}}\right]
$$


with standard deviation $\sigma$ and median radius $r_{V}$. In these simulations, the median radius $r_{V}$ and the standard deviation $\sigma$ were varied from 0.25 to $25.0 \mu \mathrm{m}$ and from 0.3 to 0.7 , respectively.
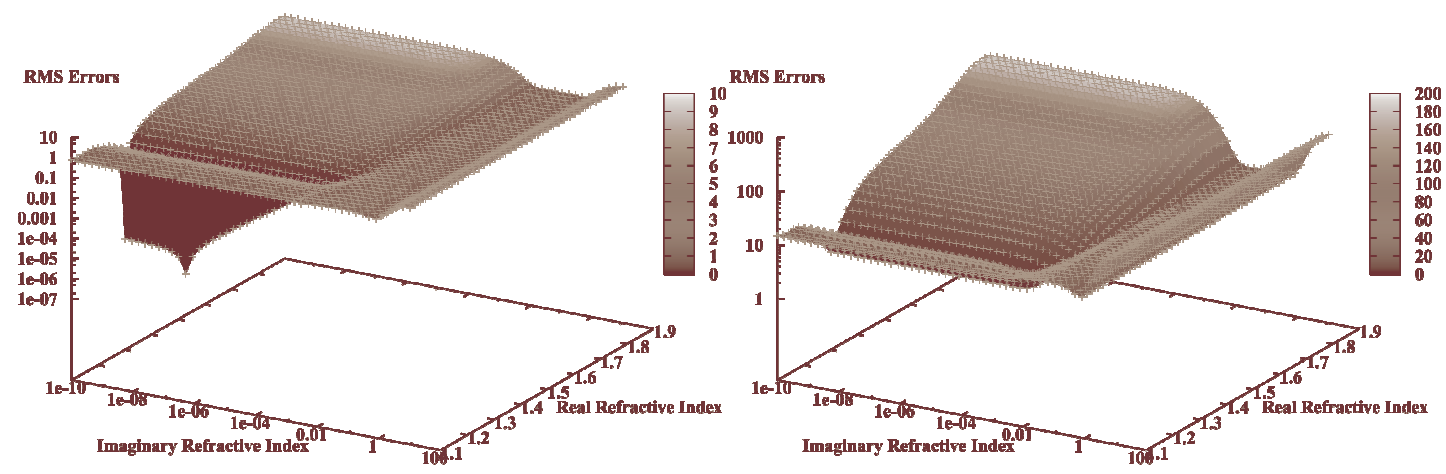

Figure 1. RMS errors functions plotted in the case of non absorbing particles $m=1.3+10^{-8} i$. Left panel is the test without measurement errors, right panel is the test with measurement errors $\varepsilon=0.1$.

Figure 1 shows the results obtained for the case of non absorbing particles. The synthetic measurements were computed for the refractive index value $m_{\text {meas }}=1.3+10^{-8} i$, $r_{V}=5 \mu \mathrm{m}$ and $\sigma=0.5$. The $R M S$ values are plotted as 3D surface, which depends on values of the real and imaginary parts of the variable refractive index $m$. The left panel corresponds to $\mathcal{E}=0$ and the right panel is for $\mathcal{E}=0.1$.

In the case without error of measurements, the data of the D2PN are very sensitive to the refractive index values. The sharp minimum is seen exactly at $m=1.3+10^{-8} i$. Measurement errors substantially affect the sensitivity. Only the range of variation of the imaginary part can be estimated, i.e., $\chi \leq 10^{-4}$. On the other hand, the data remain sensitive to the real part value.
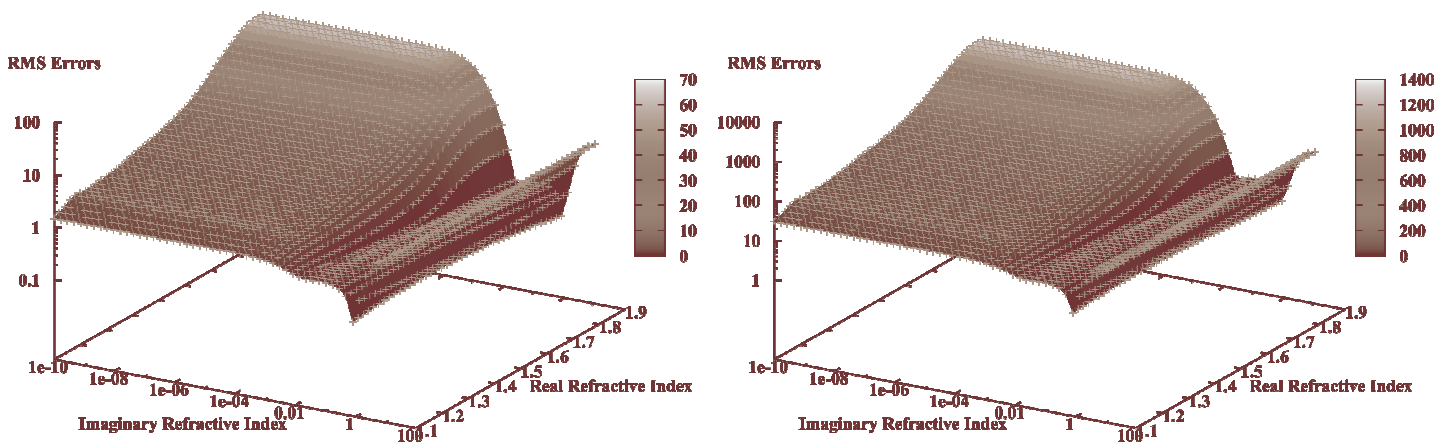

Figure 2. RMS errors functions plotted in the case of absorbing particles $m=1.3+1 i$. Left panel is the test without measurement errors, right panel is the test with measurement errors $\varepsilon=0.1$. 
In the case when the absorption of particles is very high, i.e., $\chi=1$, the data of the D2PN are not sensitive to the real part of the refractive index (see Figure 2). To the contrary, the imaginary part can be retrieved with good accuracy.

Sensitivity tests of the same kind were performed for the real and the imaginary parts ranged from 1.1 through 1.9 and from $10^{-3}$ through $10^{-1}$, respectively. It follows that in such cases the data of the D2PN provide possibility to estimate the both parts of the refractive index with quite good accuracy (measurement errors $\varepsilon=0.1$ ).

The data of the D2PN are sensitive to the microphysical parameters $\sigma$ and $r_{V}$ as well. That is, $\sigma$ and $r_{V}$ can be retrieved along with the refractive index.

Our calculations, performed for polarized scattered intensities $I_{\perp}$ and $I_{/ /}$, showed that, compared to the phase function case, the sensitivity increased, but not essentially. At the same time, it is expected that measurements of $I_{\perp}$ and $I_{/ /}$will be substantial for characterization of nonspherical particles.

\section{CONCLUSION}

- The data of the D2PN enable to retrieve microphysical parameters of spherical aerosols along with the assessment of the refractive index.

- In the case when the absorption of particles is very high, the data of the D2PN are not sensitive to the real part of the refractive index. The imaginary part can be retrieved with good accuracy.

- Measurement errors substantially reduce the sensitivity to the imaginary part in the case of non absorbing particles.

\section{Acknowledgments}

The authors are grateful to O. Dubovik for providing the flexible inverse code and the software package with kernels.

\section{References}

1. Volten, H., Muñoz, O, Hovenier, J.W., de Haan, J.F., Vassen, W., van der Zande, W.J., and Waters, L.B.F.M. WWW scattering matrix database for small mineral particles at 441.6 and $632.8 \mathrm{~nm}$. JQSRT, 90, pp. 191-206 (2005).

2. Dubovik O., "Optimization of Numerical Inversion in Photopolarimetric Remote Sensing," in Photopolarimetry in Remote Sensing, G. Videen, Y. Yatskiv and M. Mishchenko, eds. (Kluwer Academic Publishers, Dordrecht, Netherlands, 2004), pp. 65-106.

3. Dubovik, O., Sinyuk, A., Lapyonok, T., Holben, B.N., Mishchenko, M., Yang, P., Eck, T.F., Volten, H., Muñoz, O., Veihelmann, B., van der Zande, W.J., Leon, J.-F., Sorokin, M., and Slutsker, I. Application of spheroid models to account for aerosol particle nonsphericity in remote sensing of desert dust. J. Geophys. Res., 111, D11208, (2006). 